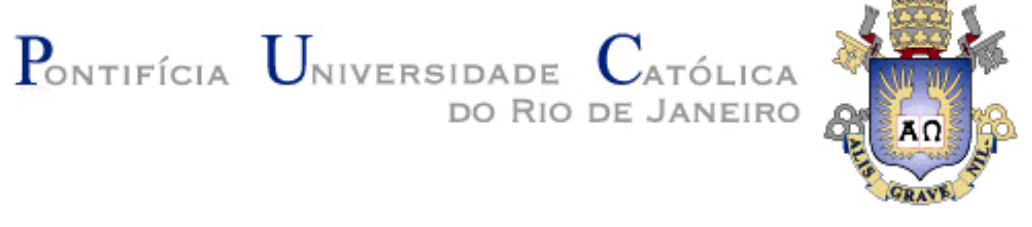

Rômulo Castello Henriques Ribeiro

\title{
APLICAÇÕES DE PROBABILIDADE E ESTATÍSTICA EM ANÁLISES GEOTÉCNICAS
}

Tese de Doutorado

Tese apresentada ao Programa de Pós-graduação em Engenharia Civil da PUC-Rio como requisito parcial para obtenção do título de Doutor em Engenharia Civil.

Orientador: Prof. Alberto S. Ferraz Jardim Sayão

Rio de Janeiro, junho de 2008 
Rômulo Castello Henriques Ribeiro

\section{APLICAÇÕES DE PROBABILIDADE E ESTATÍSTICA EM ANÁLISES GEOTÉCNICAS}

Tese apresentada como requisito parcial para obtenção do título de Doutor pelo Programa de Pósgraduação em Engenharia Civil da PUC-Rio. Aprovada pela Comissão Examinadora abaixo assinada.

\section{Prof. Alberto Sampaio Ferraz Jardim Sayão \\ Presidente/Orientador \\ Departamento de Engenharia Civil - PUC-Rio}

Prof. Celso Romanel Departamento de Engenharia Civil - PUC-Rio

Prof. João Luis Pascal Roehl Departamento de Engenharia Civil - PUC-Rio

Prof ${ }^{a}$. Ana Cristina Castro Fontenla Sieira UERJ

Prof $^{\mathrm{a}}$. Katia Vanessa Bicalho UFES

Prof. Paulo Cesar de Almeida Maia UENF

Prof. José Eugenio Leal Coordenador Setorial do Centro Técnico Científico - PUC-Rio

Rio de Janeiro, 12 de junho de 2008. 
Todos os direitos reservados. É proibida a reprodução total ou parcial do trabalho sem autorização da universidade, do autor e do orientador.

\section{Rômulo Castello Henriques Ribeiro}

Graduou-se em Engenharia Civil na UFES (Universidade Federal do Espírito Santo) em 1997. Obteve o título de mestre em Engenharia Civil (área de concentração: Geotecnia) pela PUC-Rio, em 2000. É professor da UFES e engenheiro consultor em projetos geotécnicos.

Ficha Catalográfica

Ribeiro, Rômulo Castello Henriques

Aplicações de probabilidade e estatística em análises geotécnicas / Rômulo Castello Henriques Ribeiro ; orientador: Alberto S. Ferraz Jardim Sayão. - 2008.

$161 \mathrm{f}$ : : il. ; $30 \mathrm{~cm}$

Tese (Doutorado em Engenharia Civil)Pontifícia Universidade Católica do Rio de Janeiro, Rio de Janeiro, 2008.

Inclui bibliografia

1. Engenharia civil - Teses. 2. Probabilidade. 3. Estatística. 4. Recalques. 5. Ruptura. 6. Fundações. 7. Muros de arrimo. 8. Taludes. I. Sayão, Alberto S. Ferraz Jardim. II. Pontifícia Universidade Católica do Rio de Janeiro. Departamento de Engenharia Civil. III. Título. 


\section{Agradecimentos}

A Deus, por iluminar meus caminhos.

Ao professor Alberto S. F. J. Sayão, pelo empenho na correção deste trabalho e por confiar em minhas propostas.

Ao professor, primo e amigo Reno R. Castello, pelos valiosos conselhos.

Ao CNPq, pelo apoio financeiro.

Aos meus queridos pais, Vicente e Ana Maria, pelo carinho.

Em especial, à minha esposa Rachel, que com amor, carinho e companheirismo, criou um ambiente inspirador para o desenvolvimento deste trabalho.

Em especial, ao meu filho Rafael, pelas brincadeiras, pelo sorriso, pelo cafuné, por me acordar no meio da noite pedindo suco e dormir logo em seguida, pelos passeios de bicicleta, pelos beijos, pelos abraços e por uma infinidade de outros bons momentos que surgiram no decorrer desta tese, com o nascimento do nosso amado. 


\section{Resumo}

Ribeiro, Rômulo Castello Henriques; Sayão, Alberto Sampaio Ferraz Jardim. Aplicações de probabilidade e estatística em análises geotécnicas. Rio de Janeiro, 2008. 161p. Tese de Doutorado Departamento de Engenharia Civil, Pontifícia Universidade Católica do Rio de Janeiro.

Em análises geotécnicas, previsões de deformações ou de fatores de segurança são desenvolvidas com base em métodos determinísticos, que admitem como fixos e conhecidos os parâmetros do solo ou da rocha. Entretanto, tais previsões são afetadas por incertezas provenientes da impossibilidade de reprodução das condições de campo em laboratório, da perturbação do solo devida à instalação de instrumentos, das ocorrências geomecânicas não detectadas durante a campanha de sondagens, da variabilidade inerente ao maciço, entre outras. O estudo da influência dessas incertezas sobre os cálculos determinísticos, com a possibilidade da quantificação do risco de insucesso associado a um projeto geotécnico, desenvolveu-se durante as últimas décadas com base nas teorias de probabilidade e estatística. O presente trabalho realiza uma revisão bibliográfica de conceitos básicos de probabilidade e estatística, mostrando alguns avanços da aplicação desses conceitos na engenharia geotécnica. Visando apresentar formas de estimarem-se probabilidades de recalque inadmissível ou de ruptura são realizadas análises para os seguintes casos: recalques de argila mole solicitada por aterro e de fundações superficiais em areia, estabilidade de fundação superficial em solo residual e de fundação profunda em solo sedimentar, deslizamento de um muro de arrimo e estabilidade de um talude. Com o objetivo de inferir acerca dos fatores que influenciam as estimativas probabilísticas, para cada caso são realizadas comparações entre resultados obtidos com base em diferentes métodos probabilísticos e/ou determinísticos.

\section{Palavras-chave}

Probabilidade; estatística; recalques; ruptura; fundações; muros de arrimo; taludes. 


\section{Abstract}

Ribeiro, Rômulo Castello Henriques; Sayão, Alberto Sampaio Ferraz Jardim. Aplications of Probability and Statistics in Geotechnical Analyses. Rio de Janeiro, 2008. 161p. DSc Thesis - Department of Civil Engineering, Pontifícia Universidade Católica do Rio de Janeiro.

In geotechnical analyses, forecasts of safety factors or deformations are developed on the basis of deterministics methods, that admit as fixed and known the parameters of the soil or the rock. However, such forecasts are affected by uncertainties proceeding from the reproduction impossibility of the field conditions in laboratory, of the disturbance of the soil under installation of instruments, of the not detected geomechanics occurrences during the soundings campaign, of the inherent variability to the soil, among others. The study of the influence of these uncertainties on the deterministics calculations, with the possibility of the risk quantification of failure associated with a getechnical project, developed during the last decades on the basis in theories of probability and statistics. The present work make a bibliographical revision of basic concepts of probability and statistics, showing some advances of the application of these concepts in geotechnical engineering. With the objective to show forms of computing probabilities of rupture or of inadmissible settlement are make analyses for the following cases: settlement of fill on soft clay, settlement of superficial foundations in sand, stability of superficial foundation in residual soil, stability of deep foundation in sand, stability of retaining wall and dam slope stability. With the objective to verify the factors that influence the probabilist estimates, for each case is make comparisons between results given of different probabilist and/or deterministics methods.

\section{Keywords}

Probability; statistics; settlements; rupture; foundations; retaining walls; slopes. 


\section{Sumário}

1. Introdução

2. Revisão bibliográfica 26

2.1. Introdução 26

2.2. Conceitos de probabilidade 26

2.2.1. Função de probabilidade 27

2.2.2. Momentos probabilísticos 28

2.2.3. Algumas distribuições probabilísticas 29

2.2.3.1. Distribuição normal 29

2.2.3.2. Distribuição lognormal 31

2.2.3.3. Distribuição gama 32

2.2.3.4. Distribuição qui-quadrado 32

2.2.3.5. Distribuição t de Student 33

2.3. Análise estatística 34

2.3.1. Análise gráfica da amostra 34

2.3.2. Análise aritmética da amostra 36

2.3.3. Obtenção de dados estatísticos para análises probabilísticas em estudos geotécnicos 37

2.3.4. Correções da variância devidas à variabilidade espacial do solo 39

2.3.5. Inferência estatística 42

2.4. Métodos Probabilísticos 45

2.4.1. Método do Segundo Momento de Primeira Ordem 45

2.4.2. Método das Estimativas Pontuais $\quad 47$

2.4.3. Simulação de Monte Carlo 48

2.5. Confiabilidade 49

2.6. Risco Admissível 52

2.7. Aplicações de probabilidade e estatística na geotecnia 53

2.7.1. Distribuições estatísticas de parâmetros geotécnicos 54

2.7.2. Probabilidade e estatística em análises de aterro sobre argila mole $\quad 55$ 
2.7.3. Probabilidade e estatística em análises de fundações superficiais

2.7.4. Probabilidade e estatística em análises de fundações profundas

2.7.5. Probabilidade e estatística em análises de estabilidade de muro de arrimo

2.7.6. Probabilidade e estatística em análises de estabilidade de taludes

3. Análises probabilísticas associadas a previsões de recalques

65

3.1. Introdução

65

3.2. Análise probabilística aplicada a previsões de recalques edométricos

65

3.2.1. Aplicação do Método do Segundo Momento 66

3.2.2. Aplicação do Método das Estimativas Pontuais

70

3.3. Análise probabilística aplicada a previsões de recalques imediatos de fundações superficiais apoiadas em areia

3.3.1. Análises determinísticas

74

3.3.1.1. Método de Schmertmann

74

3.3.1.2. Previsões determinísticas

77

3.3.2. Análise probabilística 78

3.4. Análise dos resultados

3.4.1. Análise dos resultados referentes a probabilidades associadas a recalques edométricos

3.4.2. Análise dos resultados referentes a probabilidades de recalques imediatos de fundações superficiais em areias e apreciação da metodologia proposta

4.2. Análise de probabilidade de ruptura de fundações superficiais

4.2.1. Probabilidades de ruptura de fundações superficiais pelo Método do Segundo Momento 
4.2.2. Probabilidades de ruptura de fundações superficiais pelo Método das Estimativas Pontuais

4.3. Análise de probabilidade de ruptura de fundações profundas

4.3.1. Previsões determinísticas dos fatores de segurança

4.3.1.1. Formulações semi-empíricas para previsão de carga última de fundações profundas

4.3.1.2. Estimativas determinísticas de fatores de segurança associados à ruptura de um solo solicitado por uma estaca isolada

4.3.2. Análises de probabilidade de ruptura de uma estaca de concreto pré-moldado instalada em um solo sedimentar

4.3.2.1. Previsões de probabilidades de ruptura de fundações profundas pelo Método do Segundo Momento

4.3.2.2. Previsões de probabilidades de ruptura de fundações profundas pelo Método das Estimativas Pontuais

4.4. Análise dos Resultados

4.4.1. Considerações sobre os resultados obtidos na análise de probabilidade de ruptura de fundações superficiais

4.4.2. Considerações sobre os resultados obtidos na análise de probabilidade de ruptura de fundações profundas

5. Análises de probabilidade de deslizamento de muro de arrimo

5.1. Introdução

5.2. Aplicação do Método do Segundo Momento de Primeira Ordem para a estimativa de probabilidade de deslizamento de um muro de arrimo

5.3. Aplicação do Método das Estimativas Pontuais para a estimativa de probabilidade de deslizamento de um muro de arrimo

5.4. Análises dos resultados e sugestão para dimensionamento de muro de arrimo com base em uma probabilidade de deslizamento admissível 
5.4.1. Análise dos resultados

5.4.2. Sugestão para dimensionamento de muro de arrimo com base em uma probabilidade de deslizamento admissível

6. Análises de probabilidade de ruptura de um talude

6.1. Introdução

6.2. Aplicação de métodos determinísticos para as estimativas dos fatores de segurança do talude

6.2.1. Métodos determinísticos de análise de estabilidade de taludes

6.2.2. Médias e variâncias para $o$ fator de segurança correspondente ao talude de jusante da barragem de Benguê

6.3. Aplicação do Método do Segundo Momento para estimativas de probabilidades de ruptura do talude

6.4. Aplicação do Método das Estimativas Pontuais para estimativas de probabilidade de ruptura do talude

6.5. Análise dos resultados

7. Conclusões

8. Referências bibliográficas

9. Apêndice 1 - Valores da função distribuição acumulada normal

10. Apêndice 2 - Cálculos dos recalques

11. Apêndice 3 - Cálculos de média e variância de FS, pelos métodos do Segundo Momento e das Estimativas Pontuais, para o muro de arrimo analisado no Capítulo 5, com diversas posições de nível d'água

12. Apêndice 4 - Cálculos de média e variância de FS, pelo método do Segundo Momento, variando-se a dimensão B para o muro de arrimo analisado no Capítulo 5 


\section{Lista de figuras}

Figura 1.1. Comparação entre duas situações com médias e distribuições de FS diferentes

Figura 2.1. Área hachurada representando a probabilidade de $\mathrm{FS} \leq 1$

Figura 2.2. Gráfico de uma distribuição gaussiana com média $\mu$ e desvio padrão $\sigma$ 30

Figura 2.3. Gráfico de uma distribuição lognormal 31

Figura 2.4. Gráfico de uma distribuição gama, com $\alpha=3$ e $\beta=1$ 32

Figura 2.5. Gráficos de uma distribuição qui-quadrado

Figura 2.6. Comparação entre a distribuição t de Student e a distribuição normal padrão

Figura 2.7. Exemplo de um histograma de uma variável aleatória

Figura 2.8. Valores usuais de probabilidade e conseqüências de ruptura, Whitman (1984)

Figura 2.9. Esquema das provas de carga, Campos (1980)

Figura 2.10. Comparação entre histograma e distribuição lognormal, Fenton e Griffiths (2002)

Figura 2.11. Esquema de muro de arrimo para análise probabilística, Falabella (2006)

Figura 2.12. Seção típica do talude, Sandroni e Sayão (1992)

Figura 2.13. Seção transversal do talude submerso, antes e após o deslizamento, Duncan (1999)

Figura 2.14. Seção transversal da barragem de Curuá-Uma, Falabella (2006)

Figura 3.1. Perfil geotécnico e carregamento (aterro), Duncan (1999) 66

Figura 3.2. Influência dos parâmetros na variância do recalque 
Figura 3.3. Gráfico para obtenção da probabilidade do recalque ser inadmissível com base no Método do Segundo Momento

Figura 3.4. Distribuição probabilística do recalque com área hachurada para a determinação da probabilidade de $\rho \geq 0,5 \mathrm{~m}$

Figura 3.5. Gráfico para obtenção da probabilidade do recalque ser inadmissível com base no Método das Estimativas Pontuais

Figura 3.6. Perfil geotécnico da área experimental, Cordeiro (2004)

Figura 3.7. Edifício típico do bairro de Jardim Camburi, Vitória-ES

Figura 3.8. Distribuição do fator de influência (Schmertmann et al., 1978)

Figura 3.9. Gráfico para determinação da probabilidade de $\rho$

$\geq 25 \mathrm{~mm}$, com $\alpha=5$

Figura 3.10. Gráfico para determinação da probabilidade de $\rho$

$\geq 25 \mathrm{~mm}$, com $\alpha=6$

Figura 3.11. Gráfico para determinação da probabilidade de $\rho$

$\geq 25 \mathrm{~mm}$, com $\alpha=10$

Figura 3.12. Comparação entre histograma e distribuições normal e lognormal, para $\alpha=5$

Figura 3.13. Comparação entre histograma e distribuições normal e lognormal, para $\alpha=6$

Figura 3.14. Comparação entre histograma e distribuições normal e lognormal, para $\alpha=10$

Figura 3.15. Probabilidades de recalque inadmissível segundo dois métodos probabilísticos

Figura 4.1. Esquema de sapata

Figura 4.2. Representação gráfica da probabilidade de ruptura

Figura 4.3. Equilíbrio limite de um elemento de fundação profunda 
Figura 4.4. Perfil geotécnico de um solo sedimentar arenoso de Vila Velha-ES

Figura 4.5. Esquema de estaca pré-moldada de concreto instalada no solo sedimentar arenoso de Vila Velha-ES

Figura 5.1. Esquema do muro de arrimo (dimensões em m),

Duncan (1999)

104

Figura 5.2. Diagramas de tensões efetivas atuantes no muro

Figura 5.3. Gráfico de variação da probabilidade de ruptura com a profundidade do nível d'água no terrapleno

Figura 5.4. Gráfico de variação da probabilidade de deslizamento com a largura da base do muro

Figura 6.1. Seção transversal da barragem de Benguê, Secretaria de Recursos Hídricos do Ceará (2000)

Figura 6.2. Envoltória de resistência obtida a partir de regressão linear

Figura 6.3. Esquema de talude com malha de pontos para pesquisa da superfície crítica de ruptura

Figura 6.4. Esquema de forças na fatia, Método de Fellenius

Figura 6.5. Esquema de forças na fatia, Método de Bishop Simplificado

Figura 6.6. Variação do fator $f_{0}$ em função do parâmetro d/L e do tipo de solo

Figura 6.7. Forças atuantes em uma fatia pelo Método de Morgenstern \& Price (1965) 


\section{Lista de tabelas}

Tabela 2.1. Coeficientes de variação de parâmetros geotécnicos

Tabela 2.2. Valores de escala de flutuação 42

Tabela 2.3. Valores de $\tau=\frac{t_{\alpha}}{\sqrt{\mathrm{n}}}$ em função de $\mathrm{n}$ e $\alpha$

Tabela 3.1. Médias e desvios padrão dos parâmetros da argila mole da baía de São Francisco, Duncan (1999) 66

Tabela 3.2. Dados para o cálculo determinístico do recalque 67

Tabela 3.3. Termos para o cálculo da variância de $\rho \quad 68$

Tabela 3.4. Valores dos recalques correspondentes às dezesseis combinações

Tabela 3.5. Resultados dos ensaios CPT realizados após a remoção do aterro, Cordeiro (2004) 73

Tabela 3.6. Valores típicos de $\alpha$, Coduto (2001) 77

Tabela 4.1. Fatores de capacidade de carga propostos na literatura geotécnica $\quad 86$

Tabela 4.2. Fatores de profundidade propostos na literatura geotécnica

Tabela 4.3. Valores de tensão de ruptura e fator de segurança obtidos pelas três soluções consideradas

Tabela 4.4. Coeficientes de variação para parâmetros geotécnicos referentes a solos residuais, Guedes (1997)

Tabela 4.5. Variância de FS com base nos coeficientes máximos de variação e nos fatores de Meyerhof (1963)

Tabela 4.6. Variância de FS com base nos coeficientes máximos de variação e nos fatores de Hansen (1970) 
Tabela 4.7. Variância de FS com base nos coeficientes máximos de variação e nos fatores de Vesic $(1973,1975)$

Tabela 4.8. Valores de FS correspondentes às oito combinações com tensões de ruptura estimadas pela solução de Meyerhof (1963)

Tabela 4.9. Valores de FS correspondentes às oito combinações com tensões de ruptura estimadas pela solução de Hansen (1970)

Tabela 4.10. Valores de FS correspondentes às oito combinações com tensões de ruptura estimadas pela solução de Vesic $(1973,1975)$

Tabela 4.11. Valores médios de FS pelo método das Estimativas Pontuais

Tabela 4.12. Variâncias de FS pelo método das Estimativas Pontuais

Tabela 4.13. Valores de k e $\alpha$, Aoki e Velloso (1975)

Tabela 4.14. Valores de F1 e F2, Aoki e Velloso (1975)

Tabela 4.15. Valores de k e $\alpha$, Laprovitera (1988)

Tabela 4.16. Valores de F1 e F2, Laprovitera (1988) e Benegas (1993)

Tabela 4.17. Valores de C, Décourt e Quaresma (1978)

Tabela 4.18. Fatores de segurança de acordo com os métodos determinísticos

Tabela 4.19. Valores de V[FS] de acordo com os métodos determinísticos de estimativa de carga última com base no Método do Segundo Momento

Tabela 4.20. Resultados das análises determinísticas, Método de Aoki e Velloso (1975)

Tabela 4.21. Resultados das análises determinísticas, Método de Aoki e Velloso com fatores k, $\alpha, F 1$ e F2 de Laprovitera (1988) e Benegas (1993)

Tabela 4.22. Resultados das análises determinísticas, Método de Décourt e Quaresma (1978) 
Tabela 4.23. Valores de V[FS] de acordo com os métodos determinísticos de estimativa de carga última com base no Método das Estimativas Pontuais

Tabela 5.1. Valores de média e desvio padrão dos parâmetros para análise de estabilidade, Duncan (1999) 102

Tabela 5.2. Cálculo da variância de FS, referente ao deslizamento do muro, com empuxos calculados pela teoria de Rankine (1857)

Tabela 5.3. Cálculo da variância de FS, referente ao deslizamento do muro, com empuxos calculados pela teoria de Coulomb (1776)

Tabela 5.4. Variação da probabilidade de deslizamento com a profundidade do nível d'água em relação à superfície do terrapleno - Método do Segundo Momento

Tabela 5.5. Resultados das análises determinísticas para o Método das Estimativas Pontuais

Tabela 5.6. Variação da probabilidade de deslizamento com a profundidade do nível d'água em relação à superfície do terrapleno - Método das Estimativas Pontuais

Tabela 5.7. Variação da probabilidade de deslizamento com a largura da base do muro

Tabela 6.1. Valores de média e variância dos parâmetros de resistência

Tabela 6.2. Valores médios dos fatores de segurança para o talude da barragem de Benguê, para a situação de ausência do N.A. no talude

Tabela 6.3. Valores médios dos fatores de segurança para o talude da barragem de Benguê em situação crítica, com nível d'água na altura máxima

Tabela 6.4. Variância de FS, utilizando-se o método de Fellenius (1936), com N.A. ausente 
Tabela 6.5. Variância de FS, utilizando-se o método de Bishop Simplificado (1955), com N.A. ausente

Tabela 6.6. Variância de FS, utilizando-se método de Janbu

Simplificado(1973), com N.A. ausente

Tabela 6.7. Variância de FS, utilizando-se o método de

Morgenstern \& Price (1965), com N.A. ausente

123

Tabela 6.8. Variância de FS, utilizando-se o método de Fellenius (1936), com N.A. máximo

Tabela 6.9. Variância de FS, utilizando-se o método de Bishop Simplificado (1955), com N.A. máximo

Tabela 6.10. Variância de FS, utilizando-se método de Janbu Simplificado (1973), com N.A. máximo

Tabela 6.11. Variância de FS, utilizando-se o método de Morgenstern \& Price (1965), com N.A. máximo

Tabela 6.12. Probabilidades de ruptura para a situação de ausência do N.A. no talude

Tabela 6.13. Probabilidades de ruptura para a situação de fluxo com nível d'água máximo

Tabela 6.14. Valores de FS para a condição de ausência de N.A. no talude

Tabela 6.15. Valores de FS para a condição de N.A. máximo no talude

Tabela 6.16. Probabilidades de ruptura, de acordo com Método das Estimativas Pontuais, para uma condição de ausência de N.A. no talude

Tabela 6.17. Probabilidades de ruptura, de acordo com Método das Estimativas Pontuais, para uma condição de N.A. máximo no talude 


\section{Lista de símbolos}

$a^{\prime}$ - intecepto da envoltória no plano $\mathrm{p}^{\prime}-\mathrm{q}^{\prime}$

$\mathrm{A}_{\mathrm{b}}$ - área da base

$\mathrm{A}_{1}$ - área lateral

b - largura da fatia

B - menor dimensão da fundação

C - fator de correlação dependente do tipo de solo, método de Décourt e

Quaresma (1978)

c'- coesão efetiva do solo

$\operatorname{Cov}($ ) - coeficiente de variação

Cc - índice de compressão

Cr - índice de recompressão

$\mathrm{cv}$ - coeficiente de adensamento vertical

CPT - cone penetration test

$d c$ - fator de profundidade dependente do ângulo de atrito e da razão D/B

$d q$ - fator de profundidade dependente do ângulo de atrito e da razão D/B

$d \gamma$ - fator de profundidade dependente do ângulo de atrito e da razão D/B

$D$ - profundidade de embutimento

DMT - Flat Dilatometer Test

$e$ - índice de vazios

E - módulo de elasticidade

E - empuxo 
$E[]$ - valor esperado

$\mathrm{f}_{\mathrm{s}}$ - resistência lateral do ensaio de cone holandês

$f_{0}$ - fator de correção empírico, método de Janbu (1955)

$f($ ) - função densidade de probabilidade

$F($ ) - função de distribuição

F1 - fator de escala e execução das estacas

F2 - fator de escala e execução das estacas

FS - fator de segurança

Gs - densidade real dos grãos

$G(X)$ - função de desempenho

$\mathrm{H}$ - espessura

$\mathrm{I}_{\varepsilon}$ - fator de influência para recalques

$\mathrm{k}$ - coeficiente de permeabilidade

$\mathrm{k}$ - fator de correlação entre $\mathrm{N}$ e qc

$\mathrm{K}_{\mathrm{a}}$ - coeficiente de empuxo ativo

$\mathrm{K}_{\mathrm{p}}$ - coeficiente de empuxo passivo

L - comprimento da estaca

n - número de determinações

$\mathrm{N}$ - força normal

$\mathrm{N}$ - número de golpes da sondagem SPT

$\mathrm{N}_{60}$ - número de golpes da sondagem SPT para uma energia igual a $60 \%$ da energia teórica

$\bar{N}_{l}$ - média dos números de golpes do SPT obtidos ao longo da superfície lateral da estaca em um intervalo $\Delta \mathrm{L}_{\mathrm{i}}$ 
$\bar{N}_{p}$ - média dos números de golpes do SPT para a zona de ponta da estaca $N_{c}$ - fator de capacidade de suporte adimensional $N_{q}$ - fator de capacidade de suporte adimensional $N_{\gamma}$ - fator de capacidade de suporte adimensional

OCR - over consolidation ratio

$P_{f}$ - probabilidade de ruptura

$\mathrm{p}^{\prime}$ - semi-soma entre tensões efetivas principais maior e menor

$q^{\prime}$ - semi-diferença entre tensões efetivas principais maior e menor

$q$ - tensão imposta ao solo pela fundação

$\mathrm{q}_{\mathrm{c}}$ - resistência de ponta do ensaio CPT

qDMT - resistência do ensaio dilatométrico

$\mathrm{q}_{\mathrm{p}}$ - tensão resistente de ponta

$\mathrm{q}_{\mathrm{li}}$ - tensão resistente lateral, considerada constante em um intervalo de comprimento $\Delta \mathrm{L}_{\mathrm{i}}$

$\mathrm{Q}_{\mathrm{r}}$ - carga última ou capacidade de carga total do sistema estaca-solo

$\mathrm{Q}_{\mathrm{p}}$ - resistência de ponta

$\mathrm{Q}_{1}$ - resistênicia lateral

R - capacidade de resistência

s - desvio padrão da amostragem

S - grau de saturação

S - demanda de solicitação

SPT - standard penetration test

Su - resistência não drenada

t( ) - função de freqüência 
T( )- função de freqüência acumulada

$\mathrm{T}$ - Força tangencial

$\mathrm{t}_{\alpha}$ - valor obtido através da função de Student

u - poro-pressão

U - perímetro da estaca

U - resultante de poro-pressões

V[ ] - variância da distribuição

$w$ - teor de umidade

W - peso

x - variável aleatória

$\bar{X}$ - média da amostragem

Z - variável aleatória padronizada

$\alpha$ - fator de correlação entre fs e qc

$\alpha$ - fator de correlação empírico entre módulo de elasticidade e resistência de ponta do cone

$\alpha$ - nível de confiança

$\alpha$ - parâmetro da função gama

$\alpha$ - ângulo de inclinação do tardoz

$\alpha^{\prime}$ - ângulo de inclinação da envoltória no plano $\mathrm{p}^{\prime}-\mathrm{q}^{\prime}$

$\beta$ - índice de confiabilidade

$\beta$ - ângulo de inclinação do terrapleno

$\delta$ - escala de flutuação

$\delta$ - ângulo de atrito solo/muro

$\Delta$ - acurácia 
$\Delta \sigma$ - acréscimo de tensão

$\Delta \mathrm{z}$ - comprimento total considerado em uma dada direção

$\varepsilon_{z}$ - deformação específica vertical

$\phi ’$ - ângulo de atrito efetivo do solo

$\phi(Z)$ - função densidade de probabilidade e distribuição acumulada aferidas em $Z$

$\gamma$ - peso específico

$\gamma_{t}$ - peso especifico total

$\gamma_{\text {nat }}$ - peso específico natural

$\Gamma(\alpha)$ - função gama

$\Gamma$ - função de variância

$\lambda$ - constante a ser determinada por processo iterativo do método de Morgenstern \& Price (1965)

$\mu$ - média da distribuição

$\mu_{G}$ - valor médio da função de desempenho

$\rho$ - recalque

$\theta$ - inclinação da base da fatia

$\sigma$ - desvio padrão

$\sigma^{\prime}$ - tensão efetiva

$\sigma_{p}^{\prime}-$ tensão de pré-adensamento

$\sigma[$ ] - desvio padrão da distribuição

$\tau$ - tensão cisalhante 